\title{
Asymmetry induces long-lasting energy current transients inside molecular loop circuits
}

\author{
Fabienne Michelini* and Katawoura Beltako ${ }^{\dagger}$ \\ Aix Marseille Université, Université Toulon, CNRS, IM2NP, Marseille, France
}

(Received 25 March 2019; published 18 July 2019)

\begin{abstract}
Energy transport and conversion at nanoscale have become an important topic of fundamental and applied research, in particular for conceiving ground-breaking solutions in energy-aware digital electronics and energy production. In this work, we propose a formal framework to address time-dependent energy transport inside quantum networks. The approach permits us to investigate how energy transferred to electrons by a femtosecond laser pulse is stored and released in a molecular circuit consisting of two donor-acceptor branches connected to an acceptor chain. Additionally, the two donors may be coupled, creating a loop inside the circuit. Time-resolved analysis reveals that when a difference exists between the two donor-acceptor branches, a loop current occurs and persists during relaxation, while only a small amount of current flows through the acceptor chain. A long-lasting energy flow thus emerges from the asymmetry of the molecular structure.
\end{abstract}

DOI: 10.1103/PhysRevB.100.024308

\section{INTRODUCTION}

Molecular-scale architectures are integrated in various applied fields including digital electronics [1,2], optoelectronics [3], photovoltaics [4], and thermoelectricity [5-7], due to the fact that they comply with several practical criteria for miniaturization of systems. Molecules offer simpler ways to achieve active systems that are functionalizable at the atomic scale, and that can be created easily and at low cost as single channels or stacked assemblies, compared to traditional components of solid-state technologies. In parallel with these human-made solutions, nature also provides biological devices and machines that could deepen our understanding of energy transport and conversion, in particular, active systems involved in photosynthesis [8-11]. It is thus hoped that deciphering how photosynthetic complexes function could reveal unexpected keys for controlling and boosting the specifications of biologically inspired technological solutions [12-14]. More particularly, during the early stages of photosynthesis, energy transport inside these complexes from absorbing chromophores to reaction centers is a long-lived process, very robust against decoherence, that exhibits efficiency above 95\% [15].

Whether they are human-made or natural, molecular systems generally form condensed-phase quantum networks, extremely sensitive to the environment [16], in which crucial processes may occur on ultra-short time scales. In nanodevices, there have been advances to simulate and discuss timeresolved energy and heat transport [17-20]. In molecular and biological systems, time-dependent modeling has proven a necessary procedure to settle on the most important steps in charge and energy control, e.g., light absorption [21-23], charge separation $[16,24]$, but also energy transfer $[25,26]$.

\footnotetext{
*fabienne.michelini@univ-amu.fr

${ }^{\dagger}$ Present address: Okinawa Institute of Science, and Technology Graduate University, Onna Son, Okinawa 9040495, Japan.
}

However, the theoretical framework of time-dependent energy transport in the quantum regime remains very challenging [27], and is still debated, especially due to the difficulty of complying with standard laws of thermodynamics formulated in the quantum regime [28-30]. Indeed, only a few works have been proposed to unravel energy fluxes inside the quantum network formed by molecular complexes, despite the fact that such studies are necessary to understand the intimate management of energy inside the systems, to give a clear picture of how they work, and finally to be able to find efficient solutions in order to evacuate or harvest, and eventually store energy. In this work, we derive a general formulation of the energy current operator inside a network whose Hamiltonian platform is quadratic in creation and annihilation operators. The resulting expression of energy current is given in terms of Green's functions, and used inside a two-branched, donoracceptor molecular network in which the two donors can connect to form a loop with the acceptor. Here, we examine how energy acquired by electrons from the interaction of donors with a femtosecond laser pulse flows inside this nanocircuit. We find that the system may durably retain energy fluxes inside the loop for slightly different donor-acceptor branches.

\section{ENERGY CURRENT INSIDE QUANTUM NETWORKS}

In quantum transport, the global system has to be divided in two parts: the central region that represents an open quantum system, eventually interacting and out of equilibrium, and reservoirs that are infinite, but generally considered as noninteracting and at equilibrium. It is thus possible to use equilibrium statistics as Bose-Einstein and Fermi-Dirac functions to describe particle occupation. Within this picture, quantum transport simulations are based on lattice Hamiltonian models of the central region. The sites of the lattice may be positions, atoms, or small molecules, etc., depending on the scale and the nature ( $a b$ initio or empirical) of the method chosen to describe the properties of the moving particles. Each model actually proposes a discrete formulation of all possible 


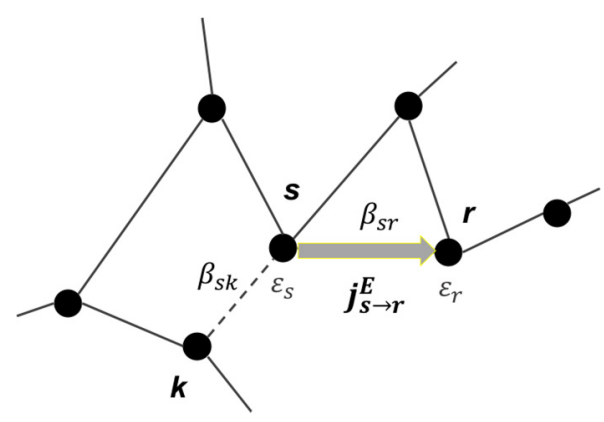

FIG. 1. Schematic network and parameter definitions.

connections between different sites $s$ of the system, which defines a quantum network. This picture can be extended to the case of interacting nanosystems using a mean-field approximation, for which the total Hamiltonian still has a quadratic form using creation and annihilation operators $d_{s}^{\dagger}$ and $d_{s}$. The network is thus modeled by an ensemble of $M$ sites of energy $\epsilon_{s}$, each interconnected to other sites $r$ with couplings $\beta_{s r}$ (for uncoupled sites $\beta_{s r}=0$ ). These couplings may be overlapping integrals, tunneling parameters, dipole-dipole interaction, or light-matter interaction parameters (see Fig. 1).

The difficulty of defining an energy current operator resides in the fact that, in contrast with the charge current operator for which the number of particles $N$ is equal to the sum of on-site numbers of particules, $N=\sum_{s} d_{s}^{\dagger} d_{s}$, the total Hamiltonian of the system does not have the form of a sum of local Hamiltonians. One has to choose a form, generally one that verifies the maximum of conservation and symmetry laws [31-34], which has never been proposed in a satisfactory manner. In Ref. [31], the authors discussed the issue and developed an energy current operator inside a one-dimensional chain. Following their work, we here address the case of a network. The network Hamiltonian is partitioned into subsystems according to $H=\sum_{s} H_{s}$ with $H_{s}=\epsilon_{s} d_{s}^{\dagger} d_{s}+$ $\frac{1}{2} \sum_{r} V(s, r)$, where $V(s, r)=\beta_{s r} d_{s}^{\dagger} d_{r}+$ H.c. is the coupling operator (H.c. stands for Hermitian conjugated). This choice implies that the half part of an intersite coupling is given to each of the two sites, so that they form two separated subsystems. For defining an energy current operator, the Heisenberg's equation of motion $i \hbar \frac{d H_{s}}{d t}=\left[H_{s}, H\right]+i \hbar \frac{\partial H_{s}}{\partial t}$ is recast in a continuity equation for the energy [35]. We have thus defined the operator of energy current flowing from site $s$ to site $r$ as

$$
j_{s \rightarrow r}^{E}=\frac{i}{\hbar}\left[H_{s}, H_{r}\right],
$$

following the derivation given in Appendix A. This proposal complies with the exchange symmetry requirement, $j_{s \rightarrow r}^{E}=$ $-j_{r \rightarrow s}^{E}$, and the energy conservation law $\dot{H}_{s}+\sum_{r} j_{s \rightarrow r}=\frac{\partial H_{s}}{\partial t}$. The commutator inside Eq. (1) is evaluated for the specific case of fermions, and it gives the energy current operator expression:

$$
\begin{aligned}
j_{s \rightarrow r}^{E}= & \frac{i}{2 \hbar}\left[\epsilon_{s} n_{s}-\epsilon_{r} n_{r}, V(s, r)\right]+\frac{i}{4 \hbar}\left\{\sum_{k} W(s, r, k)\right. \\
& +W(k, s, r)+W(s, k, r)\},
\end{aligned}
$$

where $W(x, y, z)=[V(x, y), V(y, z)]$. The expression of the energy current operator Eq. (2) is the sum of two contributions. The first contribution is related to the operator of the particle current flowing from site $s$ to site $r$. The second contribution consists of three different commutators of coupling operators involving the site $s$. These three terms are actually related to spatial current-current correlators through

$$
W(x, y, z)(t)=-\frac{\hbar^{2}}{e^{2}}\left[j_{x \rightarrow y}^{c}(t), j_{y \rightarrow z}^{c}(t)\right]
$$

in antisymmetrized form, where $j_{x \rightarrow y}^{c}=\frac{i e}{\hbar}\left[n_{x}, V(x, y)\right]$ is the usual charge current operator. These contributions to the energy current operator show the need to further deepen the analysis of different forms of current-current correlations for the study of energy [36,37]. Finally, the expression of the energy current, $J_{s r}=\left\langle j_{s \rightarrow r}^{E}\right\rangle$, is calculated as the mean value of the energy current operator, here derived in terms of nonequilibrium Green's functions [38,39]:

$$
\begin{aligned}
J_{s r}(t)= & -\frac{1}{\hbar} 2 \operatorname{Re}\left[\frac{\epsilon_{s}(t)+\epsilon_{r}(t)}{2} \beta_{r s}(t) G_{s r}^{<}(t, t)\right. \\
& +\frac{1}{4} \sum_{k}\left\{\beta_{r s}(t) G_{s k}^{<}(t, t) \beta_{k r}(t)+\beta_{s k}(t) G_{k r}^{<}(t, t) \beta_{r s}(t)\right. \\
& \left.\left.+\beta_{k s}(t) G_{s r}^{<}(t, t) \beta_{r k}(t)\right\}\right],
\end{aligned}
$$

where $G_{s r}^{<}\left(t, t^{\prime}\right)=i\left\langle d_{r}^{\dagger}\left(t^{\prime}\right) d_{s}(t)\right\rangle$ (see Appendix B for details). The expression of the energy current reveals two contributions, again. The first is proportional to the particle current flowing from site $s$ to site $r$ with the coefficient $\frac{\epsilon_{s}+\epsilon_{r}}{2}$. The second contribution involves three different types of products of intersite coupling parameters. Actually, these last three terms include nonlocal energy transport pathways inside the network, as $J_{s r}$ depends on other couplings than $\beta_{r s}$ through these terms. They are related with spatial current-current correlators, as shown by Eq. (3). Additionally, these terms also exist in the stationary regime, but they do not appear in the expression of the charge current. It might be possible to measure them from optical measurements [40]. The expression of Eq. (4) may be compared to the one derived in Ref. [41], which is often used in the stationary (st) working of nanodevices [42-44]: $J_{s r}^{\mathrm{st}}=\frac{1}{h} \operatorname{Re} \int d E E \beta_{r s} G_{s r}^{<}(E)$. The energy current $J^{\text {st }}$ was also extracted from a continuity equation, but for the mean value of the energy current, without defining an energy current operator. The strength of the derivation of $J^{\text {st }}$ is that it provides energy current conservation, which is not the case of the present proposal, a priori [31]. However, the derivation of Ref. [41] relies on the property of time translation invariance, which is at the heart of stationary problems. It is thus not possible to directly extend this derivation for time-dependent problems.

\section{TWO-BRANCHED MOLECULAR NANOCIRCUIT}

The energy current expression of Eq. (4) was used to investigate photoinduced energy currents flowing inside the molecular network represented in Fig. 2. This small network is the active region of a nanocircuit, consisting of a twobranched donor-acceptor junction in contact with electron reservoirs on each side (R), schematically represented in 
(a)

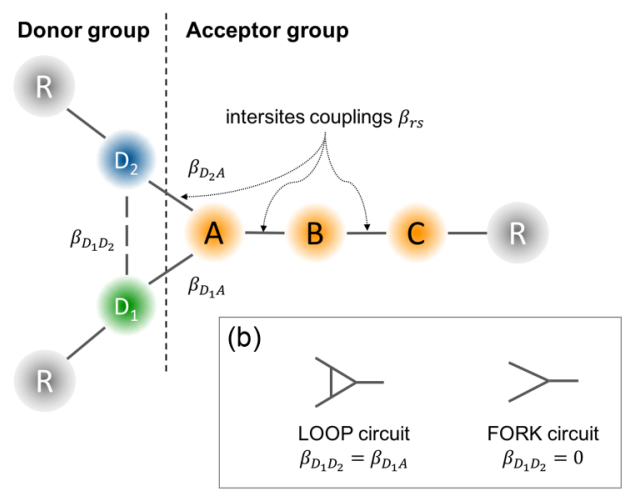

(c)

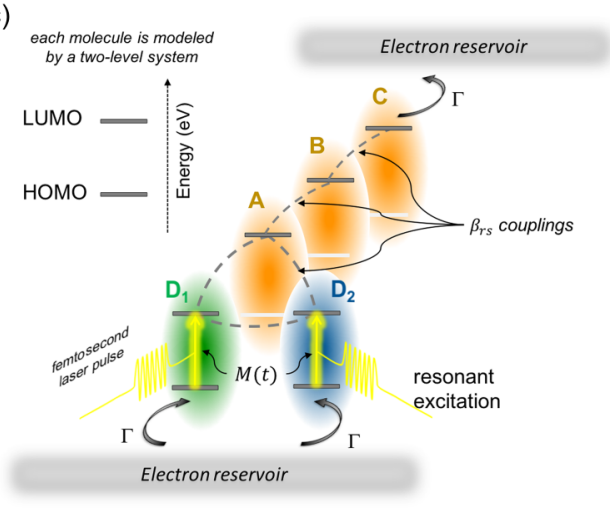

FIG. 2. (a) Molecular network. (b) Schematic molecular loop and fork circuits. (c) Level structure of the molecular network made of two donors and three acceptors in series. The two donors interact simultaneously with a resonant femtosecond laser pulse of boxcar shape. The molecular network is in contact with two wide-band electron reservoirs.

Fig. 2(a). The donor group comprises two donors, $D_{1}$ and $D_{2}$, connected to an acceptor $A$ with the two coupling parameters $\beta_{D_{1} A}$ and $\beta_{D_{2} A}$. Node $A$ is the first element of a three-site linear chain, in which the molecules, labeled $A, B$, and $C$, are taken to be identical and form the acceptor group. In the donor group, the two donors, $D_{1}$ and $D_{2}$, may or may not be connected via the coupling parameter $\beta_{D_{1} D_{2}}$, which defines two types of circuits shown in Fig. 2(b): the loop in which the two donors are coupled with the parameter $\beta_{D_{1} D_{2}}=\beta_{D_{1} A}$, and the fork, for which the two donors are disconnected, $\beta_{D_{1} D_{2}}=0$. The network level structure is represented in Fig. 2(c). Each molecule is described by a two-level system representing highest occupied molecular orbital (HOMO) and lowest unoccupied molecular orbital (LUMO) states. The HOMO states of the acceptor group are not connected to the HOMO states of the donor group, which provides $D-A$ junction rectification. In the default circuit configuration, all HOMO and LUMO levels are aligned and fixed, $\epsilon_{\mathrm{HOMO}}=-0.6 \mathrm{eV}$ and $\epsilon_{\mathrm{LUMO}}=$ $+0.6 \mathrm{eV}$, and coupling parameters between LUMO (HOMO) states are equal to the same value $\beta=0.05 \mathrm{eV}(0.2 \beta)$. Additionally, each level is broadened with a unique parameter representing a few picoseconds in order to include decoherence effects, e.g., vibrational degrees of freedom, that happen in this time scale. Finally, the two reservoirs represent regions where electrons are collected and thermalize, like metallic electrodes. These reservoirs are here treated in the wideband limit approximation [45], with $\Gamma=0.05 \mathrm{eV}$. The Fermi energy lies in the middle of the gap, $E_{F}=0$. In this nanocircuit, the energy current is induced by light absorption, as illustrated in Fig. 2(c). The two donors interact simultaneously with a femtosecond laser pulse of boxcar shape, and central pulsation of which $\omega$ is resonant with the isolated donor gap. The pulse-driven transition between the HOMO and LUMO states inside $D_{1}$ and $D_{2}$ is modeled by the same pulse-donor coupling: $M(t)=M_{0} \cos (\omega t) \Pi_{W}\left(t-t_{0}\right)$, where $\Pi_{W}$ is the boxcar function of width $W$ and starting at $t_{0}$. In the present study, $M_{0}=0.05 \mathrm{eV}, \hbar \omega=1.2 \mathrm{eV}, W=20 \mathrm{fs}$, and $t_{0}=1 \mathrm{fs}$.

Time-resolved and spectral physical quantities were numerically calculated on a cluster architecture, using the efficient technique of wave functions proposed by Gaury and co-workers in Ref. [27], here extended to the energy current from Eq. (4). We obtain

$$
\begin{aligned}
J_{s r}(t)= & \frac{2}{h} \operatorname{Im} \sum_{\alpha m_{\alpha}} \int d E f_{\alpha}(E) \times\left[\frac{\epsilon_{s}(t)+\epsilon_{r}(t)}{2} \beta_{r s}(t) \Psi_{m_{\alpha} E}(s, t) \Psi_{m_{\alpha} E}^{*}(r, t)+\frac{1}{4} \sum_{k}\left\{\beta_{r s}(t) \Psi_{m_{\alpha} E}(s, t) \Psi_{m_{\alpha} E}^{*}(k, t) \beta_{k r}(t)\right.\right. \\
& \left.\left.+\beta_{s k}(t) \Psi_{m_{\alpha} E}(k, t) \Psi_{m_{\alpha} E}^{*}(r, t) \beta_{r s}(t)+\beta_{k s}(t) \Psi_{m_{\alpha} E}(s, t) \Psi_{m_{\alpha} E}^{*}(r, t) \beta_{r k}(t)\right\}\right]
\end{aligned}
$$

where $\Psi_{m_{\alpha} E}(s, t)$ is the $s$ th component of the wave function of mode $m_{\alpha}$, and $f_{\alpha}$ the Fermi function of reservoir $\alpha$ (see Appendix $\mathrm{C}$ for details).

\section{DIRECT CURRENT IN ASYMMETRIC $D$ - $A$ CONFIGURATION}

We investigate the effects on energy current of the asymmetry between the two donor-acceptor $D_{1}-A$ and the $D_{2}-A$ branches, varying the donor-acceptor coupling parameter $\beta_{D_{2} A}$ from zero to $2 \beta_{D_{1} A}$. For $\beta_{D_{2} A}=0$, the circuit is a linear chain.
For $\beta_{D_{2} A}=\beta_{D_{1} A}$, the circuit is in its symmetric configuration, regardless of the $\beta_{D_{1} D_{2}}$ value. We examine the integral over time of the transient energy currents, here called direct energy currents, flowing between sites $D_{1}, D_{2}, A$, and $B$. Direct energy currents for the loop and the fork are presented in Fig. 3. As a global result, direct energy currents are greater in the loop than in the fork, except in the vicinity of the symmetric configuration. This observation confirms that interdonor coupling might enhance optoelectronic conversion performances in molecular architectures, as shown for smaller networks in the steady-state regime, from master equations 


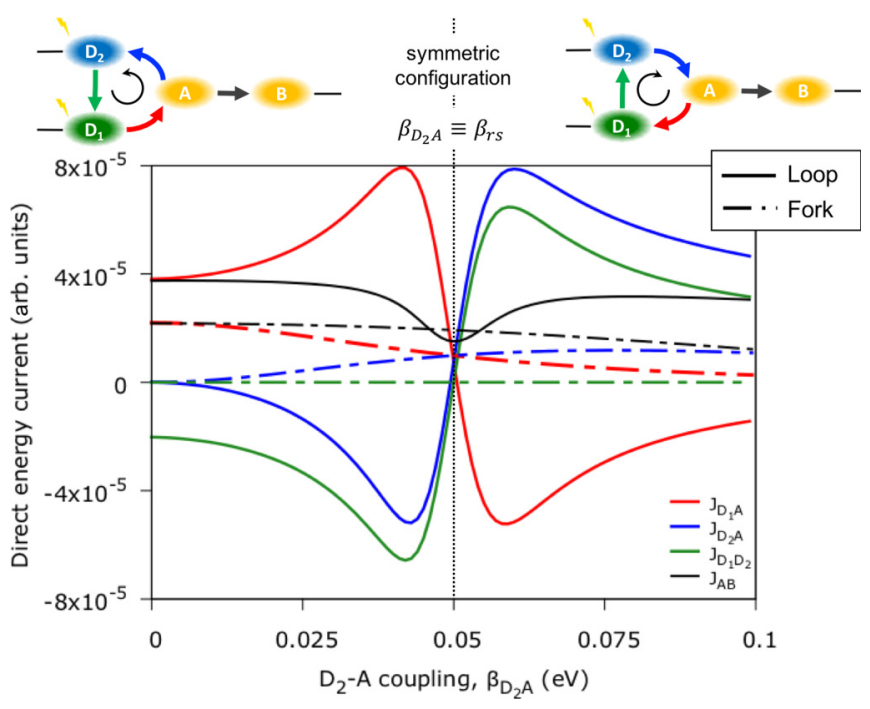

FIG. 3. Direct energy current (in arbitrary units) flowing between sites $D_{1}, D_{2}, A$, and $B$ for the loop (solid lines) and the fork (dashed lines) circuits, as a function of $D_{2}-A$ coupling.

at weak coupling to reservoir [46-48]. But in contrast with these previous studies, here, the impact of such a coherence is not systematically beneficial. The direct energy current $J_{A B}$ flowing outside the junction exhibits a dip at the symmetric configuration in the loop (when the two donors are coupled), the minimum of which is below the current value obtained in the fork case (when the two donors are not coupled).

The direct energy currents $J_{D_{1,2} A}$ flowing in the $D_{1,2}-A$ branches more strongly differ between the fork and the loop. In the fork circuit, $J_{D_{1} A}$ exhibits a monotonic decrease while $J_{D_{2} A}$ displays a monotonic increase with increasing $\beta_{D_{2} A}$ values. The two currents remain always positive, lower than $J_{A B}$, and they cross at the symmetric configuration, as expected. In the loop circuit, $J_{D_{1} A}$ increases with increasing $\beta_{D_{2} A}$, until it reaches a maximum positive value close to, but before, the symmetric configuration $\left(\beta_{D_{2} A}<\beta_{D_{1} A}\right)$. At this point, $J_{D_{1} A}$ decreases abruptly until a minimum negative value, still close to, but after, the symmetric configuration $\left(\beta_{D_{2} A}>\beta_{D_{1} A}\right)$. Then, $J_{D_{1} A}$ increases again. Direct energy currents $J_{D_{2} A}$ and $J_{D_{1} D_{2}}$ exhibit an opposite variation. These results show that, on average, energy circulates anticlockwise inside the loop for $\beta_{D_{2} A}<\beta_{D_{1} A}$, and clockwise for $\beta_{D_{2} A}>\beta_{D_{1} A}$, as illustrated at the top of Fig. 3. In the vicinity of the symmetric configuration, the loop circulation disappears: direct energy currents change sign in a small range of $\beta_{D_{2} A}$ values. At the symmetry point and as expected for symmetry reasons, energy current vanishes inside the $D_{1}-D_{2}$ link, $J_{D_{1} D_{2}}=0$, and energy current flows in parallel inside the $D_{1}-A$ and the $D_{2}-A$ branches, $J_{D_{1} A}=J_{D_{2} A}$. For $\beta_{D_{2} A}>\beta_{D_{1} A}$, the loop circulation of the energy current is restored but in the opposite direction than the one it has for $\beta_{D_{2} A}<\beta_{D_{1} A}$. In the loop, currents are globally greater than the resulting current that flows outside the junction. Finally, these results obtained for the loop $\left(\beta_{D_{1} D_{2}}=\beta\right)$ and the fork $\left(\beta_{D_{1} D_{2}}=0\right)$ at strong coupling to the reservoir also suggest that modifying the interdonor coupling has a greater impact on the current for asymmetric configurations of the molecular circuit.

\section{LONG-LASTING ENERGY CURRENT TRANSIENTS IN THE LOOP}

In order to understand direct energy current variations shown in Fig. 3, we look into the time-resolved energy currents inside the two parallel donor-acceptor branches, $J_{D_{1.2} A}$, for four $\beta_{D_{2} A}$ values. For $\beta_{D_{2} A}=0$ : Sites $D_{2}$ and $A$ are disconnected; $\beta_{D_{2} A}=0.042 \mathrm{eV}: J_{D_{1} A}$ is close to a maximum $\left(J_{D_{2} A}\right.$ a minimum); $\beta_{D_{2} A}=0.05 \mathrm{eV}$, which corresponds to the symmetric configuration: $J_{D_{1} A}$ and $J_{D_{2} A}$ nearly vanish; and finally $\beta_{D_{2} A}=0.060 \mathrm{eV}: J_{D_{1} A}$ is close to a minimum $\left(J_{D_{2} A}\right.$ a maximum). Each of these four currents is plotted as a function of time in Fig. 4 in two separated graphs, which permits us to distinguish between short-term and long-term responses of the loop. Indeed, each response divides into a driven regime, corresponding to the laser pulse application, and a relaxation regime, in which currents oscillate without external excitation. The four curves corresponding to the four $\beta_{D_{2} A}$ values are remarkably similar in the driven regimes, while strong differences exist in the relaxation regime. Indeed, relaxation oscillations hold for longer times for $\beta_{D_{2} A}=0.042$ and $0.060 \mathrm{eV}$, in contrast with the two other cases, $\beta_{D_{2} A}=0.000 \mathrm{eV}$ and $\beta_{D_{2} A}=0.050 \mathrm{eV}$. Actually, the peaks of direct energy currents shown in Fig. 3 originate from long-lived relaxation oscillations, and not enhanced amplitudes, of time-dependent energy currents. Moreover, when the whole molecule is very close to the symmetric configuration, energy current does not change sign during these long-lived oscillations. Such relaxation oscillations would nonetheless be damped by increasing decoherence throughout the spectral broadening parameter we used, or coupling to reservoirs $\Gamma$, as shown for the charge current in Ref. [23]. From these time-resolved energy currents, we thus infer that the same loop circulation observed for direct energy currents also exists at each time during relaxation, at least for $\beta_{D_{2} A}$ values taken close to the direct current peaks. After laser excitation ceases, the molecular system retains an internal energy current loop that releases a small amount of energy current to the acceptor chain at each time. This process is not allowed if the molecular loop is symmetric, $\beta_{D_{2} A}=0.05 \mathrm{eV}$, nor if it is broken, $\beta_{D_{2} A}=0$. eV. Currents inside the loop are not equal because of the displacement energy current at each node $d H_{s} / d t$, which is analogous to the displacement charge current [49], and strictly zero for stationary functioning. Additionally, we have numerically verified that $d H_{A} / d t=\left(J_{D_{1} A}+J_{D_{2} A}-\right.$ $\left.J_{A B}\right)$. At last, time-resolved energy current also reveals an intriguing property shown in the insets of Fig. 4: $J_{D_{2} A}(t)$ is nonzero for $\beta_{D_{2} A}=0$, even if it remains two orders of magnitude smaller than the other currents. Indeed, the energy current formula obtained Eq. (4) applied to the present molecular network splits into six terms, schematically represented in Fig. 5 for $J_{D_{2} A}$. It appears that all these terms vanish for $\beta_{D_{2} A}=$ 0 , except the one $(f)$ related to the product $\beta_{D_{2} D_{1}} \beta_{D_{1} A}$, and hence to the loop structure. It shows that in contrast with particle current, energy current includes additional current-current spatial correlation terms that reveal the nonlocal character of energy transfer. These nonlocal terms remain one to two orders of magnitude smaller than the direct term proportional to the charge current in regions where energy currents are important, in particular around their extremum values. 


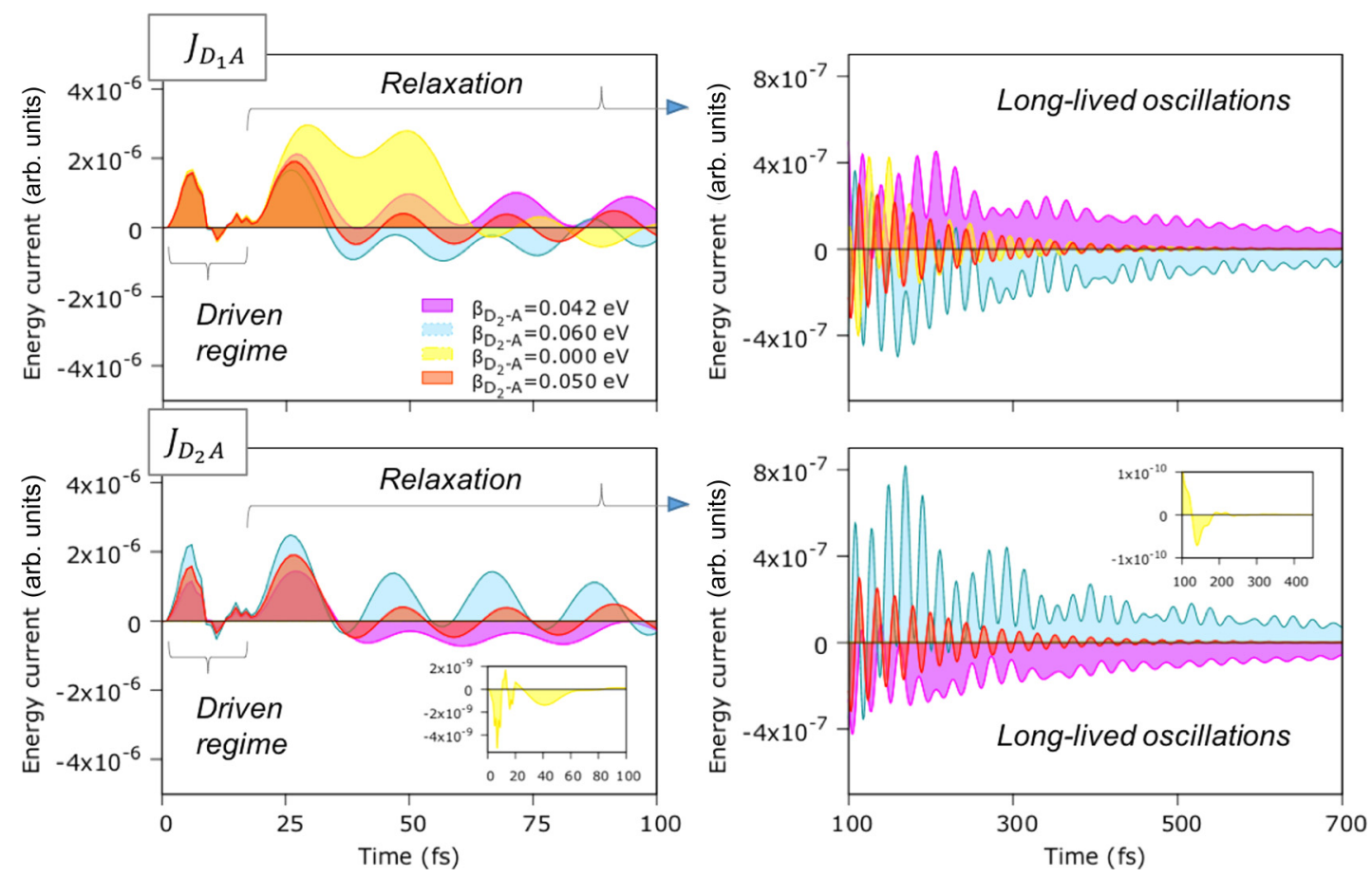

FIG. 4. Time-resolved energy currents $J_{D_{1} A}$ and $J_{D_{2} A}$ in the driven and relaxation regimes for four different $D_{2}-A$ coupling values, $\beta_{D_{2}-A}=$ 0.042 (purple), 0.060 (sky blue), 0.000 (yellow, fork circuit), and $0.050 \mathrm{eV}$ (red, symmetric loop circuit). For convenience, the results have been divided into several graphs with different scales of time and energy current. Left and right panels represent the short and long time responses, respectively. The two insets show a smaller but nonzero energy current $J_{D_{2} A}$ for $\beta_{D_{2}-A}$ equal to zero, which is discussed in the text.

\section{CONCLUSION}

This work provides a framework to discuss time-dependent energy transport inside networks. The energy current operator was derived from a local equation of energy conservation and then averaged in terms of Green's functions. The energy current is finally reformulated within the wave-function technique for numerical simulations. The dynamics of energy transfer is thus analyzed inside a two-branched donoracceptor junction connected to a molecular chain on the acceptor side. A loop can be formed inside the nanocircuit if the two donors are coupled. Following excitation of the two donors by a femtosecond laser pulse, energy currents may circulate around and around in this loop, releasing a small amount of current inside the chain during persistent
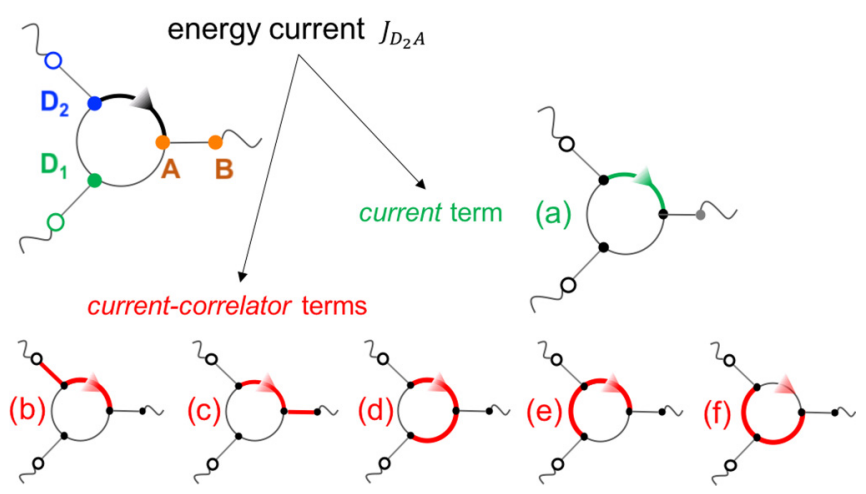

FIG. 5. Schematic representation of the different terms inside the energy current flowing from $D_{2}$ to $A$ [see Eq. (4)]. relaxation. This phenomenon occurs when the circuit is close to the perfect loop, which is provided by identical donoracceptor couplings. Indeed, this phenomenon cannot occur if the loop is perfect or if the loop is open, forming a twobranched fork, because the symmetry axis given by the chain forbids the formation of a current loop. In these two cases, the two donor-acceptor branches provide parallel channels for efficient energy release inside the acceptor chain. This work demonstrates that asymmetry and coherence in molecular architectures may conduct long-lived phenomena even for strong coupling to the rest of the molecular system. The effect could even be enhanced by taking into account a cooperative contribution of molecular vibrations inside the loop, which calls for further theoretical work.

\section{ACKNOWLEDGMENTS}

F.M. thanks M. Luisier for the fruitful discussions they had on the definition of the energy current operator.

\section{APPENDIX A: DEFINING THE ENERGY CURRENT OPERATOR}

The central region of the whole system is seen as a network described by a discrete mesh of $N$ noninteracting sites $s$ of energy $\epsilon_{s}$ connected via parameters $\beta_{s r}$ to sites $r$, called neighbors if $\beta_{s r} \neq 0$. The total Hamiltonian reads

$$
H=H_{\text {net }}+H_{\text {res }}+H_{T},
$$


with $H_{\text {res }}=H_{L}+H_{R}$ is the total reservoir Hamiltonian. With details,

$$
\begin{aligned}
H_{\mathrm{net}} & =\sum_{s} \epsilon_{s} d_{s}^{\dagger} d_{s}+\sum_{s, r} \beta_{s r} d_{s}^{\dagger} d_{r}, \\
H_{\alpha \in\{L, R\}} & =\sum_{j \in \alpha} \epsilon_{\alpha j} c_{\alpha j}^{\dagger} c_{\alpha j}, \\
H_{T} & =\sum_{\alpha=L, R} \sum_{<s, j \in \alpha>} V_{\alpha j s} c_{\alpha j}^{\dagger} d_{s}+\text { H.c., }
\end{aligned}
$$

where we have introduced the associated creation and annihilation operators $d_{s}^{\dagger}$ and $d_{s}$ for the network and $c_{\alpha j}^{\dagger}$ and $c_{\alpha j}$ for the reservoir $\alpha$ (H.c. stands for Hermitian conjugated).

The network Hamiltonian is partitioned according to

$$
H_{\text {net }}=\sum_{s} H_{s},
$$

with

$$
H_{s}=\epsilon_{s} d_{s}^{\dagger} d_{s}+\frac{1}{2} \sum_{r} \beta_{s r} d_{s}^{\dagger} d_{r}+\text { H.c. }
$$

This partition involves the definition of $N$ subsystems surrounding $s$, whose energy operator is written in a compact form:

$$
H_{s}=\epsilon_{s} n_{s}+\frac{1}{2} \sum_{r} V(s, r)
$$

introducing the coupling operator

$$
V(s, r)=\beta_{s r} d_{s}^{\dagger} d_{r}+\beta_{s r}^{*} d_{r}^{\dagger} d_{s} .
$$

The operator $V$ verifies $V(s, r)=V(r, s)$.

We form an energy current operator $j_{s \rightarrow r}^{E}$ flowing from sites $s$ to $r$ from the continuity equation for energy:

$$
\dot{H}_{s}+\sum_{r} j_{s \rightarrow r}^{E}=\frac{\partial H_{s}}{\partial t} .
$$

The Heisenberg's equation of motion of the local energy operator at site $s$ of the network is

$$
\frac{d H_{s}}{d t}=-\frac{i}{\hbar}\left[H_{s}, H\right]+\frac{\partial H_{s}}{\partial t},
$$

where the term

$$
\frac{\partial H_{s}}{\partial t}=\frac{d \epsilon_{s}}{d t} d_{s}^{\dagger} d_{s}+\frac{1}{2} \sum_{r} \frac{d \beta_{s r}}{d t} d_{s}^{\dagger} d_{r}+\text { H.c. }
$$

accounts for external energy sources, which provides energy balance. In this work, we focus on the energy current inside the network, which means that we consider sites $s$ following the condition $\left[H_{s}, H\right]=\left[H_{s}, H_{\text {net }}\right]$. From $\left[H_{s}, H_{\text {net }}\right]=$ $\sum_{r}\left[H_{s}, H_{r}\right]$, we propose to define the energy current operator as

$$
j_{s \rightarrow r}^{E}=\frac{i}{\hbar}\left[H_{s}, H_{r}\right]
$$

Developing the commutator $\left[H_{s}, H_{r}\right]$ specifically for fermions, we obtain the expression of the energy current operator given Eq. (2). The information that $k$ is a neighbor of $r$ is already included in the fact that $\beta_{r, k} \neq 0$. It is worth noting that the term $[V(s, k), V(k, r)]$ is nonzero only if the mesh contains loops (the term $[V(s, r), V(r, k)]$ may also contain loop terms). The definition of an energy current operator given Eq. (2) complies with the symmetry property:

$$
j_{s \rightarrow r}^{E}=-j_{s \rightarrow r}^{E} .
$$

\section{APPENDIX B: ENERGY CURRENT USING GREEN'S FUNCTIONS}

To investigate the functioning of nanosystems, it is convenient to define the elementary electron Green's functions:

$$
\begin{aligned}
G_{v \mu}^{\tau}\left(t, t^{\prime}\right) & =-i\left\langle T d_{v}(t) d_{\mu}^{\dagger}\left(t^{\prime}\right)\right\rangle, \\
G_{v \mu}^{<}\left(t, t^{\prime}\right) & =i\left\langle d_{\mu}^{\dagger}\left(t^{\prime}\right) d_{v}(t)\right\rangle, \\
G_{v \mu}^{>}\left(t, t^{\prime}\right) & =-i\left\langle d_{v}(t) d_{\mu}^{\dagger}\left(t^{\prime}\right)\right\rangle, \\
G_{v \mu}^{r}\left(t, t^{\prime}\right) & =-i \Theta\left(t-t^{\prime}\right)\left\langle\left\{d_{v}(t), d_{\mu}^{\dagger}\left(t^{\prime}\right)\right\}\right\rangle \\
& =\Theta\left(t-t^{\prime}\right)\left[G_{n m}^{>}\left(t, t^{\prime}\right)-G_{v \mu}^{<}\left(t, t^{\prime}\right)\right], \\
G_{v \mu}^{a}\left(t, t^{\prime}\right) & =i \Theta\left(t^{\prime}-t\right)\left\langle\left\{d_{v}(t), d_{\mu}^{\dagger}\left(t^{\prime}\right)\right\}\right\rangle \\
& =\Theta\left(t^{\prime}-t\right)\left[G_{n m}^{<}\left(t, t^{\prime}\right)-G_{v \mu}^{>}\left(t, t^{\prime}\right)\right],
\end{aligned}
$$

where $\tau$ stands for time ordered on the Keldysh contour [38]. The fermion time ordering operation $T$ is defined by

$$
T\left[f\left(t^{\prime}\right) g(t)\right]=\Theta\left(t^{\prime}-t\right) f\left(t^{\prime}\right) g(t)-\Theta\left(t-t^{\prime}\right) g(t) f\left(t^{\prime}\right),
$$

where $\Theta\left(t^{\prime}-t\right)$ is the Heaviside function.

We deduce the expression of the energy current given Eq. (4) as the mean value of the energy current operator evaluated in terms of Green's functions.

\section{Remarks.}

a. Generalization. The present derivation is the same for sites in contact or inside a reservoir. The difference lies in the expression of the Green's functions $G_{v \mu}^{<}$, if one of the sites $v$ or $\mu$ is in the reservoir. It is then necessary to solve the differential equation for $G_{v \mu}^{<}$; see, e.g., Ref. [45].

b. One dimensional case. We have verified that we recover the formula obtained in the one-dimensional case by $\mathrm{Wu}$ and Segal in Ref. [31]. For $r=s-1$,

$$
\begin{aligned}
j_{s \rightarrow s-1}^{E}= & \frac{i}{\hbar} \times\left\{\frac{1}{2}\left[\epsilon_{s} n_{s}-\epsilon_{s-1} n_{s-1}, V(s, s-1)\right]\right. \\
& +\frac{1}{4}\{[V(s, s-1), V(s-1, s-2)] \\
& +[V(j=s+1, s), V(s, s-1)] \\
& +\underbrace{\sum_{j}[V(s, j), V(j, s-1)]}_{0 \text { because } j \text { does not exist }}\},
\end{aligned}
$$


and for $r=s+1$,

$$
\begin{aligned}
j_{s \rightarrow s+1}^{E}= & \frac{i}{\hbar} \times\left\{\frac{1}{2}\left[\epsilon_{s} n_{s}-\epsilon_{s+1} n_{s+1}, V(s, s+1)\right]\right. \\
& +\frac{1}{4}\{[V(s, s+1), V(s+1, s+2)] \\
& +[V(s-1, s), V(s, s+1)] \\
& +\underbrace{\sum_{j}[V(s, j), V(j, s-1)]}_{0, \text { because } j \text { does not exist }}\}\} .
\end{aligned}
$$

In Ref. [31], the authors define

$$
j_{s}^{E}=j_{s \rightarrow s+1}^{E} .
$$

In the general case, the mesh may contain nodes and loops, and one has to define a convention for incoming and outgoing fluxes inside the infinitesimal volume surrounding the point $s$.

\section{APPENDIX C: ENERGY CURRENT USING WAVE FUNCTIONS}

The wave-function (WF) technique was proposed by Gaury and co-workers as an efficient numerical implementation of quantum transport calculations. At the present time, it is still restricted to noninteracting nanosystems, or, as in the present case, for interacting nanosystems treated in mean-field approximation. Reference [27] gives a detailed presentation of the technique; here we provide the basics to introduce the energy current in terms of wave functions. Additionally, all the information about Green's functions used here for timedependent transport may be found in Ref. [38].

The starting point is to deal with a time-independent reservoir Hamiltonian, which is always possible to obtain by gauge transformation. In such a framework, the reservoir self-energy may be recast into

$$
\Sigma_{\text {res }}^{<}\left(t-t^{\prime}\right)=i \sum_{\alpha} \int \frac{d E}{2 \pi} f_{\alpha}(E) e^{-i(E / \hbar)\left(t-t^{\prime}\right)} \Gamma_{\alpha}(E)
$$

( $\hbar=1$ in Ref. [27]). The idea of this technique is to use the eigenvectors of $\Gamma_{\alpha}(E)$ as the minimal information to grasp the mean values of physical quantities, like current, through projections of the Green's functions onto this basis. It is thus needed:

$$
\Gamma_{\alpha}(E)=\sum_{m_{\alpha}} v_{m_{\alpha}}(E) \chi_{m_{\alpha} E} \chi_{m_{\alpha} E}^{\dagger},
$$

where $\chi_{m_{\alpha} E}$ are the transverse mode $m_{\alpha}$ coming from the reservoir $\alpha$ and $v_{m_{\alpha}}(E)$ is its associated velocity.
All the Green's functions are expressed using and defining the wave functions $\Psi_{m_{\alpha} E}(t)$ as follows. From the Keldysh's transport equation,

$$
G^{\lessgtr}\left(t, t^{\prime}\right)=\iint d t_{1} d t_{2} G^{r}\left(t, t_{1}\right) \Sigma_{\mathrm{res}}^{\lessgtr}\left(t_{1}, t_{2}\right) G^{a}\left(t_{2}, t^{\prime}\right),
$$

the lesser and greater components of the Green's functions may be reformulated,

$$
\begin{aligned}
& G^{<}\left(t, t^{\prime}\right)=i \sum_{\alpha m_{\alpha}} \int \frac{d E}{2 \pi} f_{\alpha}(E) \Psi_{m_{\alpha} E}(t) \Psi_{m_{\alpha} E}^{\dagger}\left(t^{\prime}\right), \\
& G^{>}\left(t, t^{\prime}\right)=-i \sum_{\alpha m_{\alpha}} \int \frac{d E}{2 \pi}\left[1-f_{\alpha}(E)\right] \Psi_{m_{\alpha} E}(t) \Psi_{m_{\alpha} E}^{\dagger}\left(t^{\prime}\right),
\end{aligned}
$$

by defining the wave functions as

$$
\Psi_{m_{\alpha} E}(t)=\sqrt{v_{m_{\alpha}}(E)} \int d u G^{r}(t, u) e^{-i E u} \xi_{m_{\alpha} E} .
$$

Then, from the general formula,

$$
G^{r}\left(t, t^{\prime}\right)=\theta\left(t-t^{\prime}\right)\left[G^{>}\left(t, t^{\prime}\right)-G^{<}\left(t, t^{\prime}\right)\right],
$$

is deduced

$$
G^{r}\left(t, t^{\prime}\right)=-i \theta\left(t-t^{\prime}\right) \sum_{\alpha, m_{\alpha}} \int \frac{d E}{2 \pi} \Psi_{m_{\alpha} E}(t) \Psi_{m_{\alpha} E}^{\dagger}\left(t^{\prime}\right) .
$$

Gaury and co-authors showed in Ref. [27] that $\Psi$ is determined from a Schrödinger-like equation involving a source term (the last one in the right-hand side of the following equation):

$$
\begin{aligned}
i \partial_{t} \Psi_{m_{\alpha} E}(t)= & H(t) \Psi_{m_{\alpha} E}(t)+\int d u \Sigma_{\text {res }}^{r}(t-u) \Psi_{m_{\alpha} E}(u) \\
& +\sqrt{v_{m_{\alpha}}(E)} e^{-i E t} \xi_{m_{\alpha} E},
\end{aligned}
$$

which is directly derived from the equation of evolution of $G^{r}\left(t, t^{\prime}\right)$ :

$$
\begin{aligned}
i \hbar \partial_{t} G^{r}\left(t, t^{\prime}\right)= & \mathbb{I} \delta\left(t-t^{\prime}\right)+H(t) G^{r}\left(t, t^{\prime}\right) \\
& +\int d t_{1} \Sigma_{\text {res }}^{r}\left(t, t_{1}\right) G^{r}\left(t_{1}, t^{\prime}\right) .
\end{aligned}
$$

Details for efficient practical implementations can be also found in Ref. [27]. This technique allows the user to manipulate $M$ vectors of size $N$ representing all the wave functions, instead of $N \times N$ matrices representing all the Green's functions, with generally $M<N$.

Combining Eqs. (4) and (C4), one obtains the expression of the energy current in terms of wave functions provided in Eq. (5). We used this formula to numerically determine the energy currents inside the studied molecular network. 
[1] C. Joachim and M. A. Ratner, Molecular electronics: Some views on transport junctions and beyond, Proc. Natl. Acad. Sci. USA 102, 8801 (2005).

[2] D. Xiang, X. Wang, C. Jia, T. Lee, and X. Guo, Molecular-scale electronics: From concept to function, Chem. Rev. 116, 4318 (2016)

[3] M. Galperin and A. Nitzan, Molecular optoelectronics: The interaction of molecular conduction junctions with light, Phys. Chem. Chem. Phys. 14, 9421 (2012).

[4] A. Hagfeldt and M. Grätzel, Molecular photovoltaics, Acc. Chem. Res. 33, 269 (2000).

[5] Q. Zhang, Y. Sun, W. Xu, and D. Zhu, Organic thermoelectric materials: Emerging green energy materials converting heat to electricity directly and efficiently, Adv. Mater. 26, 6829 (2014).

[6] G. Benenti, G. Casati, K. Saito, and R. S. Whitney, Fundamental aspects of steady-state conversion of heat to work at the nanoscale, Phys. Rep. 694, 1 (2017).

[7] R. Miao, H. Xu, M. Skripnik, L. Cui, K. Wang, K. G. L. Pedersen, M. Leijnse, F. Pauly, K. Wärnmark, E. Meyhofer, P. Reddy, and H. Linke, Influence of quantum interference on the thermoelectric properties of molecular junctions, Nano. Lett. 18, 5666 (2018).

[8] G. D. Scholes, G. R. Fleming, A. Olaya-Castro, and R. van Grondelle, Lessons from nature about solar light harvesting, Nat. Chem. 3, 763 (2011)

[9] D. Gerster, J. Reichert, H. Bi, J. V. Barth, S. M. Kaniber, A. W. Holleitner, I. Visoly-Fisher, S. Sergani, and I. Carmeli, Photocurrent of a single photosynthetic protein, Nat. Nanotechnol. 7, 673 (2012)

[10] J.-L. Brédas, E. H. Sargent, and G. D. Scholes, Photovoltaic concepts inspired by coherence effects in photosynthetic systems, Nat. Mater. 16, 35 (2017).

[11] D. A. Cherepanov, I. V. Shelaev, F. E. Gostev, M. D. Mamedov, A. A. Petrova, A. V. Aybush, V. A. Shuvalov, A. Y. Semenova, and V. A. Nadtochenko, Mechanism of adiabatic primary electron transfer in photosystem I: Femtosecond spectroscopy upon excitation of reaction center in the far-red edge of the $\mathrm{q}_{\mathrm{Y}}$ band, Biochim. Biophys. Acta, Bioenerg. 1858, 895 (2017).

[12] A. J. White, U. Peskin, and M. Galperin, Coherence in charge and energy transfer in molecular junctions, Phys. Rev. B 88, 205424 (2013).

[13] N. H. C. Lewis, N. L. Gruenke, T. A. A. Oliver, M. Ballottari, R. Bassi, and G. R. Fleming, Observation of electronic excitation transfer through light harvesting complex II using two-dimensional electronic - vibrational spectroscopy, J. Phys. Chem. Lett. 7, 4197 (2016).

[14] E. Romero, V. I. Novoderezhkin, and R. van Grondelle, Quantum design of photosynthesis for bio-inspired solar-energy conversion, Nature (London) 543, 355 (2017).

[15] G. S. Engel, T. R. Calhoun, E. L. Read, T.-K. Ahn, T. Mancal, Y.-C. Cheng, R. E. Blankenship, and G. R. Fleming, Evidence for wavelike energy transfer through quantum coherence in photosynthetic systems, Nature (London) 446, 782 (2007).

[16] E. Zerah-Harush and Y. Dubi, Universal origin for environmentassisted quantum transport in exciton transfer networks, J. Phys. Chem. Lett. 9, 1689 (2018).

[17] A. Crépieux, F. Šimkovic, B. Cambon, and F. Michelini, Enhanced thermopower under a time-dependent gate voltage, Phys. Rev. B 83, 153417 (2011).
[18] A. Crépieux, F. Šimkovic, B. Cambon, and F. Michelini, Erratum: Enhanced thermopower under a time-dependent gate voltage [Phys. Rev. B 83, 153417 (2011)], Phys. Rev. B 89, 239907(E) (2014).

[19] A.-M. Daré and P. Lombardo, Time-dependent thermoelectric transport for nanoscale thermal machines, Phys. Rev. B 93, 035303 (2016).

[20] T. Lehmann, A. Croy, R. Gutièrrez, and G. Cuniberti, Timedependent framework for energy and charge currents in nanoscale systems, Chem. Phys. 514, 176 (2018).

[21] Y. Selzer and U. Peskin, Transient dynamics in molecular junctions: Picosecond resolution from dc measurements by a laser pulse pair sequence excitation, J. Phys. Chem. C 117, 22369 (2013).

[22] M. Galperin, Photonics and spectroscopy in nanojunctions: A theoretical insight, Chem. Soc. Rev. 46, 4000 (2017).

[23] K. Beltako, F. Michelini, N. Cavassilas, and L. Raymond, Dynamical photo-induced electronic properties of molecular junctions, J. Chem. Phys. 148, 104301 (2018).

[24] G. Candiotto, A. Torres, K. Mazon, and L. G. C. Rego, Charge generation in organic solar cells: Interplay of quantum dynamics, decoherence, and recombination, J. Phys. Chem. C 121, 23276 (2017).

[25] A. Ishizaki, T. R. Calhoun, G. S. Schlau-Cohen, and G. R. Fleming, Quantum coherence and its interplay with protein environments in photosynthetic electronic energy transfer, Phys. Chem. Chem. Phys. 12, 7319 (2010).

[26] F. Fassioli, A. Olaya-Castro, and G. D. Scholes, Coherent energy transfer under incoherent light conditions, J. Phys. Chem. Lett. 3, 3136 (2012).

[27] B. Gaury, J. Weston, M. Santin, M. Houzet, C. Groth, and X. Waintal, Numerical simulations of time-resolved quantum electronics, Phys. Rep. 534, 1 (2014).

[28] H. Hossein-Nejad, E. J. O. Reilly, and A. Olaya-Castro, Work, heat and entropy production in bipartite quantum systems, New J. Phys. 17, 075014 (2015).

[29] M. F. Ludovico, L. Arrachea, M. Moskalets, and D. Sánchez, Periodic energy transport and entropy production in quantum electronics, Entropy 18, 419 (2016).

[30] P. Strasberg, G. Schaller, T. Brandes, and M. Esposito, Quantum and Information Thermodynamics: A Unifying Framework Based on Repeated Interactions, Phys. Rev. X 7, 021003 (2017).

[31] L.-A. Wu and D. Segal, Energy flux operator, current conservation and the formal Fourier's law, J. Phys. A: Math. Theor. 42, 025302 (2009).

[32] M. F. Ludovico, J. S. Lim, M. Moskalets, L. Arrachea, and D. Sánchez, Dynamical energy transfer in ac-driven quantum systems, Phys. Rev. B 89, 161306(R) (2014).

[33] M. Esposito, M. A. Ochoa, and M. Galperin, Nature of heat in strongly coupled open quantum systems, Phys. Rev. B 92, 235440 (2015).

[34] F. Michelini, A. Crépieux, and K. Beltako, Entropy production in photovoltaic- thermoelectric nanodevices from the nonequilibrium Green's function formalism, J. Phys.: Condens. Matter 29, 175301 (2017).

[35] C. Caroli, R. Combescot, P. Nozières, and D. Saint-James, Direct calculation of the tunneling current, J. Phys. C: Solid State Phys. 4, 916 (1971). 
[36] A. Crépieux and F. Michelini, Mixed, charge and heat noises in thermoelectric nanosystems, J. Phys.: Condens. Matter 27, 015302 (2015).

[37] R. Zamoum, M. Lavagna, and A. Crépieux, Nonsymmetrized noise in a quantum dot: Interpretation in terms of energy transfer and coherent superposition of scattering paths, Phys. Rev. B 93, 235449 (2016).

[38] H. Haug and A. P. Jauho, Quantum Kinetics in Transport and Optics of Semi-Conductors (Springer, Berlin, 1996).

[39] H. Bruus and K. Flensberg, Many-body Quantum Theory in Condensed Matter Physics: An Introduction (Oxford University Press, New York, 2004).

[40] P. Février and J. Gabelli, Tunneling time probed by quantum shot noise, Nat. Commun. 9, 4949 (2018).

[41] R. Lake and S. Datta, Energy balance and heat exchange in mesoscopic systems, Phys. Rev. B 46, 4757 (1992).

[42] R. Rhyner and M. Luisier, Atomistic modeling of coupled electron-phonon transport in nanowire transistors, Phys. Rev. B 89, 235311 (2014).

[43] M. G. Pala and A. Cresti, Increase of self-heating effects in nanodevices induced by surface roughness: A full-quantum study, J. Appl. Phys. 117, 084313 (2015).
[44] M. Bescond, D. Logoteta, F. Michelini, N. Cavassilas, T. Yan, A. Yangui, M. Lannoo, and K. Hirakawa, Thermionic cooling devices based on resonant-tunneling algaas/gaas heterostructure, J. Phys.: Condens. Matter 30, 064005 (2018).

[45] A.-P. Jauho, N. S. Wingreen, and Y. Meir, Time-dependent transport in interacting and noninteracting resonant-tunneling systems, Phys. Rev. B 50, 5528 (1994).

[46] C. Creatore, M. A. Parker, S. Emmott, and A. W. Chin, Efficient Biologically Inspired Photocell Enhanced by Delocalized Quantum States, Phys. Rev. Lett. 111, 253601 (2013).

[47] K. E. Dorfman, D. V. Voronine, S. Mukamel, and M. O. Scully, Photosynthetic reaction center as a quantum heat engine, Proc. Natl. Acad. Sci. USA 110, 2746 (2013).

[48] Y. Zhang, S. Oh, F. H. Alharbi, G. S. Engel, and S. Kais, Delocalized quantum states enhance photocell efficiency, Phys. Chem. Chem. Phys. 17, 5743 (2015).

[49] W.-T. Lai, D. M. T. Kuo, and P.-W. Li, Transient current through a single germanium quantum dot, Physica E 41, 886 (2009). 